\title{
EFICIÊNCIA DE UTILIZAÇÃO DE ÁGUA E NUTRIENTES EM PLANTAS DE FEIJÃO- -DE-CORDA IRRIGADAS COM ÁGUA SALINA EM DIFERENTES ESTÁDIOS DE DESENVOLVIMENTO ${ }^{1}$
}

\author{
CLAUDIVAN F. DE LACERDA ${ }^{2}$, ANTONIA L. R. NEVES ${ }^{3}$, FRANCISCO V. A. \\ GUIMARÃES ${ }^{4}$, FRANCISCO L. B. DA SILVA ${ }^{5}$, JOSÉ T. PRISCO ${ }^{6}$, HANS R. GHEYI
}

\begin{abstract}
RESUMO: O objetivo deste trabalho foi investigar os efeitos do uso de água salina em diferentes estádios de desenvolvimento de plantas de feijão-de-corda sobre a eficiência de utilização de água e de nutrientes. $\mathrm{O}$ experimento foi conduzido no campo e obedeceu ao delineamento em blocos ao acaso, com cinco tratamentos e cinco repetições. Os tratamentos empregados foram: T1 - água de poço com condutividade elétrica $(\mathrm{CEa}) \mathrm{de} 0,8 \mathrm{dS} \mathrm{m}^{-1}$ durante todo o ciclo; T2 - água salina (CEa de $5,0 \mathrm{dS} \mathrm{m}^{-1}$ ) durante todo o ciclo; T3, T4 e T5 - água salina de 0 a 22 dias após o plantio (DAP), de 23 a 42 DAP e de 43 a 62 DAP, respectivamente. As plantas dos tratamentos T3, T4 e T5 foram irrigadas com água de poço nas demais fases do ciclo. Aos 8; 23; 43 e 63 DAP, as plantas foram colhidas e determinaram-se a matéria seca total e de grãos, a eficiência no uso da água, considerando a produção de matéria seca total $\left(E U A_{P}\right)$ e a produção de grãos $\left(E A_{G R}\right)$, e a eficiência de utilização de nutrientes $(\mathrm{K}, \mathrm{Ca}, \mathrm{N}, \mathrm{P}, \mathrm{Fe}, \mathrm{Cu}, \mathrm{Zn}$ e $\mathrm{Mn})$. A aplicação de água salina durante todo o ciclo (T2) reduziu a EUA $A_{P}$ e a $E_{\text {U }}$, enquanto a aplicação de água na fase inicial do ciclo (T3) reduziu a EUA $_{\mathrm{GR}}$ e a eficiência de utilização da maioria dos nutrientes. Por outro lado, a irrigação com água salina dos 23 aos 42 DAP (T4) e dos 43 aos 62 DAP (T5) não afetou as eficiências nos usos de água e de nutrientes.
\end{abstract}

PALAVRAS-CHAVE: Vigna unguiculata, salinidade, eficiência no uso de água, eficiência de utilização de nutrientes.

\section{WATER AND NUTRIENT USE EFFICIENCY IN COWPEA IRRIGATED WITH SALINE WATER AT DIFFERENT GROWTH STAGES}

ABSTRACT: The objective of this paper was to evaluate the effect of the saline water applied at different development stages of cowpea plants, on water and nutrient use efficiency. The experiment was set up in the field, during the dry season. A completely randomized block design, with five treatments and five repetitions was adopted. The treatments studied were: T1 (groundwater with electrical conductivity $(\mathrm{ECW})$ of $0.8 \mathrm{dS} \mathrm{m}^{-1}$ ) during the whole crop cycle; T2 saline water $\left(\mathrm{ECw}=5.0 \mathrm{dS} \mathrm{m}^{-1}\right)$ during the whole crop cycle; $\mathrm{T} 3, \mathrm{~T} 4$ and $\mathrm{T} 5$ - saline water from 0 to 22 days after sowing (DAS), from 23 to 42 DAS and from 43 to 62 DAS, respectively. The plants of T3, T4 and T5 were irrigated with groundwater in the remaining stages of the crop cycle. At 8, 23, 43 and 63 DAS the plants were harvested and the total dry mass and grain yield were measured. The water use efficiency was estimated by using total dry mass production (WUE $\mathrm{F}_{\mathrm{P}}$ ) and grain yield ( $\mathrm{WUE}_{\mathrm{GR}}$ ), as well as the nutrients use efficiency - NUE, $(\mathrm{K}, \mathrm{Ca}, \mathrm{N}, \mathrm{P}, \mathrm{Fe}, \mathrm{Cu}, \mathrm{Zn}$ and $\mathrm{Mn})$. The application of saline water during whole the crop cycle (T2) reduced $\mathrm{WUE}_{\mathrm{P}}$ and $\mathrm{WUE}_{\mathrm{GR}}$, while the use of saline water during the germination and initial plant development (T3) caused reduction in $\mathrm{WUE}_{\mathrm{GR}}$ and in the NUE for most of the analyzed nutrients. Irrigation with saline water from 23 to 42 DAS (T4) and from 43 to 62 DAS (T5) did not affect water and nutrient use efficiencies.

KEYWORDS: Vigna unguiculata, salinity, water use efficiency, nutrient use efficiency.

\footnotetext{
${ }^{1}$ Extraído da Dissertação de Mestrado da segunda autora.

${ }^{2}$ Professor Doutor, Departamento de Engenharia Agrícola, UFC, Fortaleza - CE, Fone: (0XX85) 3366.9756, cfeitosa@ufc.br

${ }^{3}$ Mestre em Irrigação e Drenagem, UFC, Fortaleza-CE, leilaneves7@ @otmail.com

${ }^{4}$ Doutor, Gerente do Laboratório de Solo e Água, Departamento de Ciências do Solo, UFC, Fortaleza - CE, valderez@ufc.br

${ }^{5}$ Estudante de Graduação em Agronomia, UFC, Fortaleza - CE, Bolsista PIBIC/CNPq, leandro_custela@ hotmail.com

${ }^{6}$ Doutor, Professor Emérito da UFC, Departamento de Bioquímica e Biologia Molecular, jtprisco@uol.com.br

${ }^{7}$ Doutor, Professor Titular, Departamento de Engenharia Agrícola, UFCG, Campina Grande - PB, hans@ deag.ufcg.edu.br

Recebido pelo Conselho Editorial em: 7-5-2008
}

Aprovado pelo Conselho Editorial em: 2-6-2009

Eng. Agríc., Jaboticabal, v.29, n.2, p.221-230, abr./jun. 2009 


\section{INTRODUÇÃO}

A irrigação é uma das tecnologias aplicadas na agricultura que mais tem contribuído para o aumento na produção de alimentos. No entanto, o uso inadequado dessa técnica vem causando problemas de degradação de solos, sendo a salinização um dos principais problemas encontrados atualmente nas áreas irrigadas, notadamente nas regiões áridas e semiáridas (GHEYI, 2000; MURTAZA et al., 2006). O uso da irrigação no semiárido brasileiro é necessário devido à pequena quantidade e à má distribuição das chuvas, porém tem causado problemas de salinidade em função dos elevados teores de sais nas fontes de água, associados ao manejo inadequado da irrigação e à falta e/ou à deficiência de drenagem. Isso tem ocasionado redução no crescimento e na produtividade das culturas, além do abandono de lotes de terra nos perímetros irrigados da região (GHEYI, 2000; DANTAS et al., 2002).

A utilização de águas salinas tem sido um problema, visto que promove o acúmulo de sais na zona radicular e reduz a produtividade da maioria das culturas (AYERS \& WESTCOT, 1999). Em função disso, recomenda-se aplicar essas águas em quantidades que superam a demanda hídrica da cultura, adicionando-se uma fração de lixiviação que reduz o acúmulo de sais na zona radicular das plantas (AYERS \& WESTCOT, 1999; ASSIS JÚNIOR et al., 2007). O aumento da lâmina de água aplicada, entretanto, causa redução na eficiência do uso da água e pode também promover a lixiviação de nutrientes, contribuindo para reduzir a produtividade e aumentar os riscos de contaminação dos lençóis subterrâneos de água (ANDRADE et al., 2008). Deve-se, também, ressaltar o fato de que o menor crescimento das plantas irrigadas com água salina pode reduzir não somente a eficiência de uso de água, como também resultar em menor extração de nutrientes pelas culturas, reduzindo a eficiência de utilização dos nutrientes aplicados (GRATTAN \& GRIEVE, 1999; LACERDA, 2005).

As alternativas para minimizar esse problema do uso de água salina na irrigação consistem na utilização de espécies ou cultivares mais tolerantes, na utilização dessas fontes de água nos estádios de maior tolerância das culturas, na mistura de água de diferentes qualidades, no uso cíclico de fontes de água com diferentes concentrações salinas, além de diversas práticas de manejo do sistema solo-planta (OSTER et al., 1984; GLENN et al., 1998; SHARMA \& RAO, 1998; MALASH et al., 2005; MURTAZA et al., 2006; CHAUHAN \& SINGH, 2008). Dentre essas estratégias, o uso de água salina apenas nos estádios de maior tolerância pode contribuir para a redução do uso de água de melhor qualidade e do acúmulo de sais no solo, limitando os impactos sobre o ambiente e sobre o desenvolvimento e as eficiências de uso de água e de nutrientes pelas culturas.

Em função do exposto acima, este trabalho teve como objetivo avaliar a eficiência no uso da água e de nutrientes $(\mathrm{K}, \mathrm{Ca}, \mathrm{N}, \mathrm{P}, \mathrm{Fe}, \mathrm{Cu}, \mathrm{Zn}$ e $\mathrm{Mn}$ ) por plantas de feijão-de-corda irrigadas com água salina aplicada nos seus diferentes estádios de desenvolvimento.

\section{MATERIAL E MÉTODOS}

O experimento foi conduzido no campo, durante a estação seca do ano de 2006 (outubro a dezembro), em área de Argissolo Vermelho-Amarelo localizada no Laboratório de Hidráulica e Irrigação da Universidade Federal do Ceará, Câmpus do Pici, em Fortaleza - CE (345'S; $\left.38^{\circ} 33^{\prime} \mathrm{W}\right)$. De acordo com a classificação de Köeppen, a área do experimento está localizada numa região de clima Aw'.

Sementes de feijão-de-corda [Vigna unguiculata (L) Walp.], cultivar Epace 10, foram semeadas, e oito dias após o plantio (DAP) foi feito o desbaste, deixando-se duas plantas por cova. As plantas foram cultivadas em espaçamento de $0,8 \mathrm{~m}$ entre linhas e $0,3 \mathrm{~m}$ entre plantas, com a densidade de plantio de 83.333 plantas por hectare, sendo submetidas aos seguintes tratamentos: T1 (testemunha) - plantas irrigadas com água de poço com condutividade elétrica (CEa) em torno de $0,8 \mathrm{dS} \mathrm{m}^{-1}$ durante todo o ciclo; T2 - água salina com CEa de 5,0 dS m${ }^{-1}$, com aplicação iniciada após a germinação e permanecendo até o final do ciclo; T3 - aplicação de água salina da semeadura 
até 22 dias após o plantio (DAP), correspondendo às fases de germinação e crescimento inicial, e água de poço no restante do ciclo; T4 - água salina aplicada de 23 a 42 DAP (fase de intenso crescimento vegetativo até a pré-floração) e água de poço nas demais fases do ciclo; T5 - água de poço da semeadura até 42 dias após o plantio e água salina aplicada a partir de 43 DAP (floração e frutificação). Para o preparo das soluções salinas, foram utilizados os sais de $\mathrm{NaCl}, \mathrm{CaCl}_{2} 2 \mathrm{H}_{2} \mathrm{O}$ e $\mathrm{MgCl}_{2} 6 \mathrm{H}_{2} \mathrm{O}$, na proporção de 7:2:1 (base equivalente), obedecendo-se à relação entre CEa e sua concentração ( mmol $\left._{\mathrm{c}} \mathrm{L}^{-1}=\mathrm{CEa} 10\right)$, conforme RHOADES et al. (2000).

O delineamento experimental utilizado foi o em blocos ao acaso, com cinco tratamentos e cinco repetições, gerando, assim, área com 25 parcelas, sendo que cada parcela teve comprimento de $5,0 \mathrm{~m}$ e largura de $3,2 \mathrm{~m}$, com quatro linhas de plantio. A parcela útil consistia em 56 plantas localizadas nas duas fileiras centrais, sendo descartada uma cova em cada extremidade dessas fileiras.

A água foi aplicada em sulcos nivelados e fechados, e as lâminas de irrigação foram definidas com base nos valores de evapotranspiração de referência (ETo), obtidas por meio do tanque Classe A e dos coeficientes da cultura (Kc), recomendados por SOUZA et al. (2005), adicionando-se a fração de lixiviação de 13\%, calculada de acordo com AYERS \& WESTCOT (1999). O turno de rega utilizado foi de três dias, sendo as plantas de todos os tratamentos irrigadas até 62 DAP. A lâmina total de água aplicada em todos os tratamentos, ao longo do ciclo, foi de 326,3 $\mathrm{mm}$.

$\mathrm{Na}$ adubação das plantas, utilizaram-se $1,1 \mathrm{~g}$ de ureia, $8 \mathrm{~g}$ de superfosfato simples e $1,5 \mathrm{~g}$ de cloreto de potássio por cova, correspondendo às doses de $20 ; 60$ e $30 \mathrm{~kg} \mathrm{ha}^{-1}$ de $\mathrm{N}, \mathrm{P}_{2} \mathrm{O}_{5}$ e $\mathrm{K}_{2} \mathrm{O}$, respectivamente (FERNANDES, 1993). As doses de ureia e superfosfato simples foram aplicadas por ocasião do plantio, enquanto a dose de potássio foi aplicada metade no plantio e metade 30 DAP. Os adubos foram aplicados em sulcos de $5 \mathrm{~cm}$ de profundidade e distanciados de $5 \mathrm{~cm}$ das plantas.

Por ocasião do desbaste (oito dias após o plantio) e aos 23; 43 e 63 dias após o plantio (DAP), grupos de plantas (20 plantas no desbaste, quatro nas coletas intermediárias e oito na coleta final) em competição plena, em cada tratamento, foram coletados, separando-se folhas (limbos foliares) e hastes (ramos e pecíolos). Após a obtenção de massa fresca, amostras homogêneas de aproximadamente $200 \mathrm{~g}$ de folhas e hastes foram acondicionadas em sacos de papel e, após secas em estufa a $60{ }^{\circ} \mathrm{C}$, foram pesadas para a obtenção da matéria seca. A produção de matéria seca foi obtida multiplicando-se a produção de massa fresca pelo teor de matéria seca das diferentes partes da planta. No final do ciclo, também foram coletados os frutos secos, separando-se os pericarpos e grãos.

As eficiências de uso de água foram calculadas, relacionando a produção primária (matéria seca total) e a lâmina total aplicada $\left(E U A_{P}\right)$, e a produção de grãos e a lâmina total de água $\left(E_{\mathrm{GR}}\right)$, sendo expressas em $\mathrm{kg}$ de matéria seca produzida/mm de água aplicada. Também foi calculada a eficiência de uso de água $\left(E U A_{P}\right)$ com base na produção primária, nos diferentes estádios, considerando a matéria seca produzida e a lâmina de água aplicada em cada estádio.

As amostras secas obtidas no final do ciclo (limbos foliares, hastes, pericarpos e grãos) foram trituradas em moinho tipo Wiley e acondicionadas em sacos de papel devidamente identificados, sendo esse o material utilizado nas determinações dos teores dos elementos minerais $(\mathrm{N}, \mathrm{K}, \mathrm{Ca}, \mathrm{P}$, $\mathrm{Fe}, \mathrm{Cu}, \mathrm{Zn}$ e $\mathrm{Mn}$ ), de acordo com as metodologias descritas por MALAVOLTA et al. (1997).

Com os dados de produção de matéria seca, de densidade de plantio e dos teores de minerais, calculou-se o total de cada elemento mineral extraído pela planta. As eficiências na utilização de nutrientes foram calculadas, relacionando a produção primária (matéria seca total) e a extração total de nutrientes $\left(E U N_{P}\right)$, e a produção de grãos e a extração total de nutrientes pelas plantas $\left(E U N_{G R}\right)$, sendo ambas expressas em $\mathrm{kg}$ de matéria seca produzida $/ \mathrm{kg}$, no caso dos macronutrientes, ou por grama (g), no caso dos micronutrientes extraídos. Também foi calculada a eficiência agronômica de 
utilização dos adubos nitrogenados, fosfatados e potássicos, relacionando a produção de grãos com a quantidade de nutrientes aplicados (FAGERIA, 1998).

Os resultados foram submetidos à análise de variância, utilizando-se do programa SAEG/UFV.

\section{RESULTADOS E DISCUSSÃO}

\section{Eficiência no uso da água}

A contribuição da água salina para a lâmina total de irrigação variou de 0 a $92,1 \%$ nos diferentes tratamentos (Tabela 1). As plantas que foram continuamente irrigadas com água salina após a germinação (T2) receberam 92,1\% de água salina, enquanto os tratamentos T1, T3, T4 e T5 receberam $0 ; 26,7 ; 33,9$ e 39,4\% de água salina, respectivamente. A aplicação de água salina nas fases de intenso crescimento (T4) e na fase reprodutiva (T5) permitiu maior economia de água de boa qualidade, sem causar impacto negativo na produtividade de grãos pela cultura (Tabela 2). Isso demonstra a eficiência dessa estratégia de uso de águas de diferentes qualidades, levando em conta a tolerância de cada estádio de desenvolvimento da cultura (MURTAZA et al., 2006).

As produtividades estimadas para o feijão-de-corda no presente estudo estão de acordo com os resultados de estudos realizados no Brasil (FREIRE FILHO et al., 2005) e na África (ADEKALU \& OKUNADE, 2006), sob condições de irrigação. Porém, são inferiores aos obtidos nas regiões produtoras dos Estados Unidos (DADSON et al., 2005). Por exemplo, em algumas regiões do Estado da Califórnia, é comum a obtenção de produções em torno de $7.000 \mathrm{~kg} \mathrm{ha}^{-1}$ (SANDEN, 1993 citado por DADSON et al., 2005). As elevadas produtividades nessas regiões devem-se, em parte, às menores temperaturas noturnas, visto que as temperaturas nas noites durante a floração aumentam a abscisão de flores, sendo esse o principal fator para a redução no número de vagens por planta (NIELSEN \& HALL, 1984). Essas diferenças podem estar associadas, também, às diferenças nas condições edafoclimáticas, incluindo a duração do dia, e nas cultivares utilizadas.

TABELA 1. Contribuição das águas de poço e salina para a lâmina total de irrigação dos diferentes tratamentos. Contribution of the well and saline water in the total irrigation depth of the different treatments.

\begin{tabular}{cccc}
\hline Tratamentos & Água do Poço $(\mathrm{mm})$ & Água Salina $(\mathrm{mm})$ & Lâmina Total $(\mathrm{mm})$ \\
\hline 1 & $326,3(100)^{1}$ & $0,0(0)$ & 326,3 \\
2 & $25,9(7,9)$ & $300,4(92,1)$ & 326,3 \\
3 & $239,2(73,3)$ & $87,1(26,7)$ & 326,3 \\
4 & $215,8(66,1)$ & $110,5(33,9)$ & 326,3 \\
5 & $197,6(60,6)$ & $128,7(39,4)$ & 326,3
\end{tabular}

${ }^{1}$ Valores entre parênteses representam os percentuais em relação à lâmina total aplicada.

TABELA 2. Matéria seca total (MST), matéria seca de grãos (MSGR) e eficiência no uso da água considerando a produção de matéria seca total $\left(E\left(A_{P}\right)\right.$ e a produção de grãos $\left(E A_{G R}\right)$ ao final do ciclo da cultura. Total dry mass (TDM), grains dry mass (GDM) and water use efficiency considering the total of dry mass produced $\left(W U E_{P}\right)$ and the grain yield $\left(W_{\mathrm{UER}}\right)$ at the end of the crop cycle.

\begin{tabular}{ccccc}
\hline Tratamentos & MST $\left(\mathrm{kg} \mathrm{ha}^{-1}\right)$ & MSGR $\left(\mathrm{kg} \mathrm{ha}^{-1}\right)$ & EUA $_{\mathrm{P}}\left(\mathrm{kg} \mathrm{MS} \mathrm{mm}^{-1}\right)$ & EUA $_{\mathrm{GR}}\left(\mathrm{kg} \mathrm{MS} \mathrm{mm}^{-1}\right)$ \\
\hline 1 & $4.526,4 \mathrm{a}^{\mathrm{l}}$ & $1.864,5 \mathrm{a}$ & $13,9 \mathrm{a}$ & $5,7 \mathrm{a}$ \\
2 & $2.586,3 \mathrm{~b}$ & $984,8 \mathrm{~b}$ & $7,9 \mathrm{~b}$ & $3,0 \mathrm{~b}$ \\
3 & $3.956,1 \mathrm{a}$ & $1.241,4 \mathrm{~b}$ & $12,1 \mathrm{a}$ & $3,8 \mathrm{~b}$ \\
4 & $4.524,3 \mathrm{a}$ & $1.827,3 \mathrm{a}$ & $13,9 \mathrm{a}$ & $5,6 \mathrm{a}$ \\
5 & $4.693,9 \mathrm{a}$ & $1.877,4 \mathrm{a}$ & $14,4 \mathrm{a}$ & $5,8 \mathrm{a}$ \\
\hline
\end{tabular}

${ }^{1}$ Médias seguidas de mesma letra nas colunas não diferem estatisticamente, pelo teste de Tukey $(P \geq 0,05)$. $n=5$. 
É importante destacar que o feijão-de-corda apresenta ampla variabilidade genética, podendo-se encontrar, nas diferentes regiões do mundo, grandes diferenças nas produtividades das cultivares em função dos seus potenciais genéticos, das condições edafoclimáticas e das condições de cultivo (DADSON et al., 2005; FREIRE FILHO et al., 2005; AJEIGBE et al., 2006). Vale salientar que as cultivares da região Nordeste do Brasil foram selecionadas em cultivo de sequeiro com emprego de pouca tecnologia. Portanto, maiores produtividades poderiam ser obtidas mediante a seleção de cultivares com o emprego de nível tecnológico mais avançado (FREIRE FILHO et al., 2005).

A aplicação de água salina nos períodos de intenso crescimento (T4) e nas fases de floração e frutificação (T5) não afetou o crescimento vegetativo e a produção de grãos pelas plantas (Tabela 3) em relação à irrigação somente com água de poço (T1), resultando na manutenção das eficiências de utilização de água, calculadas tanto com base na produção primária (MST) como na base de produção de interesse agronômico (MSGR) (Tabelas 2 e 3). A aplicação contínua de água salina reduziu tanto a MST como a MSGR, implicando redução tanto da $\mathrm{EUA}_{\mathrm{P}}$ como na da $\mathrm{EUA}_{\mathrm{GR}}$. Por outro lado, as plantas irrigadas com água salina durante os processos de germinação e crescimento inicial (T3) apresentaram redução de 33\% na MSGR, porém não tiveram a MST afetada, resultando na redução da $\mathrm{EUA}_{\mathrm{GR}}$, mas não da $\mathrm{EUA}_{\mathrm{P}}$.

TABELA 3. Eficiência no uso da água considerando a produção primária (EUA P nos diferentes estádios de crescimento. Water use efficiency considering the primary production $\left(W_{U E}\right)$ at the different growth stages.

\begin{tabular}{cccc}
\hline \multirow{2}{*}{ Tratamentos } & \multicolumn{3}{c}{ EUAp (kg MS produzida por mm de água aplicada) } \\
\cline { 2 - 4 } & $0 \mathrm{a} 22 \mathrm{DAP}$ & $23 \mathrm{a} 42 \mathrm{DAP}$ & $43 \mathrm{a} 63 \mathrm{DAP}$ \\
\hline 1 & $2,09 \mathrm{aC}$ & $12,6 \mathrm{aB}$ & $22,94 \mathrm{aA}$ \\
2 & $1,54 \mathrm{aC}$ & $5,98 \mathrm{bB}$ & $13,92 \mathrm{bA}$ \\
3 & $1,28 \mathrm{aC}$ & $7,89 \mathrm{bB}$ & $23,10 \mathrm{aA}$ \\
4 & $2,36 \mathrm{aC}$ & $12,41 \mathrm{aB}$ & $23,04 \mathrm{aA}$ \\
5 & $2,56 \mathrm{aC}$ & $13,41 \mathrm{aB}$ & $23,23 \mathrm{aA}$ \\
\hline
\end{tabular}

${ }^{\mathrm{T}}$ Médias seguidas da mesma letra minúscula nas colunas e da mesma letra maiúscula nas linhas não diferem estatisticamente, pelo teste de Tukey $(P \geq 0,05) . n=5$.

A salinidade aplicada na fase inicial do cultivo (T3) retardou o desenvolvimento da planta, sendo esse processo de retardamento já observado em estudo com essa espécie durante a fase de plântula (SOUSA et al., 2003). Além disso, observou-se, nesse tratamento, alteração no padrão de desenvolvimento da cultura e na partição de fotoassimilados, ocorrendo redução apenas na produção de frutos ao final do ciclo. Alterações na morfofisiologia de plantas de feijão-de-corda induzidas pela salinidade também têm sido observadas em outros estudos (LACERDA et al., 2006; WILSON et al., 2006).

Os resultados também demonstram que os estádios de germinação e de estabelecimento da cultura foram mais sensíveis ao acúmulo de sais na zona radicular do feijão-de-corda (MURILLOAMADOR et al., 2006). Por outro lado, MAAS \& POSS (1989) observaram redução no crescimento vegetativo, tanto quando o estresse foi aplicado durante o crescimento como durante os estádios de floração e enchimento da vagem, embora as maiores reduções tenham sido verificadas quando a cultura foi irrigada durante o primeiro estádio. De acordo com MAAS (1993), muitas culturas são suscetíveis à injúria durante a emergência do solo e durante o crescimento inicial. Uma vez estabelecidas, as plantas aumentam a tolerância durante os estádios de crescimento seguintes.

Comparando-se a eficiência do uso de água, com base na produção primária, nos diferentes estádios analisados (Tabela 3), verifica-se que a EUAP aumentou ao longo do ciclo da cultura em todos os tratamentos, atingindo valor cerca de 10 vezes maior na fase final (43 a 63 DAP) do que na fase inicial do cultivo ( 0 a 22 DAP). Isso reflete a curva de crescimento da cultura, sendo resultado comumente observado também em outras espécies (TAIZ \& ZEIGER, 2002). A análise dos diferentes tratamentos revela que os valores de EUAP não diferiram significativamente na fase 
inicial (0 a 22 DAP), embora os valores tenham sido menores em T2 e T3. Entretanto, os valores de EUA $_{P}$ decresceram significativamente nesses dois tratamentos, em relação ao $\mathrm{T} 1$, no estádio de intenso crescimento (23 a 42 DAP). Porém, na fase final, apenas o T2 apresentou valores inferiores ao T1. Isso mostra que as plantas de T3 apresentaram recuperação da sua taxa de incorporação de matéria seca na fase final do ciclo da cultura, visto que as mesmas estavam sendo irrigadas com água do poço desde 23 DAP.

\section{Eficiência de utilização de nutrientes}

A eficiência na utilização de nutrientes, representada pela relação entre a produção de grãos e a extração total de nutrientes pelas plantas $\left(E U N_{G R}\right)$, foi afetada significativamente pelos tratamentos empregados, com exceção de $\mathrm{Mn}$ e $\mathrm{Cu}$ (Tabelas 4 e 5). No entanto, a eficiência de utilização de nutrientes, representada pela relação entre a produção de matéria seca total e a extração total de nutrientes $\left(E N_{P}\right)$, praticamente não diferiu entre os tratamentos.

TABELA 4. Eficiência de utilização de macronutrientes considerando a produção de matéria seca total $\left(E U N_{P}\right)$ e de grãos $\left(E N_{G R}\right)$ em plantas de feijão-de-corda irrigadas com água salina em diferentes estádios de crescimento. Macronutrients use efficiency considering the total of dry mass produced $\left(\mathrm{NUE}_{\mathrm{P}}\right)$ and the grain yield $\left(\mathrm{NUE}_{\mathrm{GR}}\right)$ in cowpea plants irrigated with saline water at different growth stages.

\begin{tabular}{|c|c|c|c|c|c|}
\hline \multirow{2}{*}{ Elementos } & \multicolumn{5}{|c|}{ Tratamentos } \\
\hline & 1 & 2 & 3 & 4 & 5 \\
\hline & \multicolumn{5}{|c|}{ EUN $_{P}(\mathrm{~kg}$ MS produzida por kg de nutriente extraído) } \\
\hline $\mathrm{K}$ & $47,59 a b^{1}$ & $53,14 \mathrm{a}$ & $42,20 \mathrm{~b}$ & $51,08 \mathrm{a}$ & $48,45 \mathrm{ab}$ \\
\hline $\mathrm{Ca}$ & $128,91 \mathrm{a}$ & $87,22 \mathrm{c}$ & $92,08 \mathrm{c}$ & $104,29 \mathrm{bc}$ & $122,71 \mathrm{ab}$ \\
\hline $\mathrm{P}$ & $436,16 \mathrm{a}$ & $517,14 \mathrm{a}$ & $456,42 \mathrm{a}$ & $504,13 \mathrm{a}$ & $501,34 \mathrm{a}$ \\
\hline \multirow[t]{2}{*}{$\mathrm{N}$} & $50,41 \mathrm{a}$ & $44,72 \mathrm{a}$ & $41,91 \mathrm{a}$ & $44,27 \mathrm{a}$ & $44,46 \mathrm{a}$ \\
\hline & \multicolumn{5}{|c|}{$\mathrm{EUN}_{\mathrm{GR}}$ (kg de grãos produzidos por kg de nutriente extraído) } \\
\hline $\mathrm{K}$ & $20,04 \mathrm{a}$ & $20,53 \mathrm{a}$ & $13,34 \mathrm{~b}$ & $20,73 \mathrm{a}$ & $19,30 \mathrm{a}$ \\
\hline $\mathrm{Ca}$ & $55,55 \mathrm{a}$ & $33,72 \mathrm{bc}$ & $29,22 \mathrm{c}$ & $49,10 \mathrm{ab}$ & $42,69 \mathrm{ab}$ \\
\hline $\mathrm{P}$ & $182,72 \mathrm{a}$ & $197,96 \mathrm{a}$ & $143,32 \mathrm{~b}$ & $202,66 \mathrm{a}$ & $198,42 \mathrm{a}$ \\
\hline $\mathrm{N}$ & $21,19 \mathrm{a}$ & $17,13 \mathrm{ab}$ & $13,31 \mathrm{~b}$ & $17,82 \mathrm{ab}$ & $17,65 \mathrm{ab}$ \\
\hline
\end{tabular}

${ }^{1}$ Médias seguidas de mesma letra nas colunas não diferem estatisticamente, pelo teste de Tukey $(P \geq 0,05)$. $n=5$.

A otimização da eficiência nutricional é de grande importância na produção das culturas anuais, devido ao custo dos fertilizantes, imprescindíveis para o aumento da produtividade. De modo geral, a eficiência nutricional pode ser medida pelas eficiências de absorção e de utilização, sendo que, nos cálculos, pode ser considerada a produção primária ou a produção de interesse agronômico (FAGERIA, 1998).

No presente estudo, constatou-se que as plantas dos tratamentos T1, T2, T4 e T5 apresentaram comportamentos semelhantes em relação à eficiência de utilização de K, N e P (Tabela 4), Fe, Mn, $\mathrm{Cu}$ e $\mathrm{Zn}$ (Tabela 5), quando se considerou a relação entre a produção de grãos e os totais de nutrientes extraídos pelas plantas $\left(\mathrm{EUN}_{\mathrm{GR}}\right)$. Isso sugere que as plantas que foram irrigadas com água salina durante todo o ciclo (T2) apresentaram ajuste entre o crescimento, a produção e a extração de nutrientes, de modo que a eficiência de utilização se manteve semelhante ao tratamento irrigado com água de baixa salinidade. Os tratamentos T4 e T5 também não diferiram do T1, indicando que a aplicação de água salina na fase anterior à floração e na fase de formação e desenvolvimento das vagens não interfere na eficiência nutricional da cultura. Por outro lado, as plantas do $\mathrm{T} 3$ apresentaram valores inferiores para a $\mathrm{EUN}_{\mathrm{GR}}$ da maioria dos nutrientes, o que se deve à baixa produção de grãos dessas plantas (Tabela 2). 
TABELA 5. Eficiência de utilização de micronutrientes considerando a produção de matéria seca total $\left(E U N_{P}\right)$ e de grãos $\left(E N_{\mathrm{GR}}\right)$ em plantas de feijão-de-corda irrigadas com água salina em diferentes estádios de desenvolvimento. Micronutrients use efficiency considering the total of dry mass produced $\left(\mathrm{NUE}_{\mathbf{P}}\right)$ and the grain yield $\left(\mathrm{NUE}_{\mathbf{G R}}\right)$ in cowpea plants irrigated with saline water at different growth stages.

\begin{tabular}{crrrrr}
\hline \multirow{2}{*}{ Elementos } & \multicolumn{5}{c}{ Tratamentos } \\
\cline { 2 - 6 } & 1 & 2 & 3 & 4 & 5 \\
$\mathrm{Fe}$ & $4,05 \mathrm{a}^{1}$ & EUN $_{\mathrm{P}}(\mathrm{kg}$ MS produzida por g de nutriente extraído) \\
$\mathrm{Mn}$ & $12,64 \mathrm{a}$ & $3,52 \mathrm{a}$ & $4,13 \mathrm{a}$ & $4,21 \mathrm{a}$ & $4,24 \mathrm{a}$ \\
$\mathrm{Cu}$ & $76,35 \mathrm{a}$ & $87,22 \mathrm{a}$ & $9,93 \mathrm{a}$ & $9,56 \mathrm{a}$ & $10,65 \mathrm{a}$ \\
$\mathrm{Zn}$ & $24,63 \mathrm{a}$ & $23,65 \mathrm{a}$ & $93,90 \mathrm{a}$ & $82,97 \mathrm{a}$ & $90,75 \mathrm{a}$ \\
$\mathrm{Fe}$ & $1,73 \mathrm{a}$ & $1,36 \mathrm{ab}$ & $1,31 \mathrm{~b}$ & $1,72 \mathrm{a}$ & $1,70 \mathrm{a}$ \\
$\mathrm{Mn}$ & $5,51 \mathrm{a}$ & $3,41 \mathrm{a}$ & $3,17 \mathrm{a}$ & $3,88 \mathrm{a}$ & $4,27 \mathrm{a}$ \\
$\mathrm{Cu}$ & $31,96 \mathrm{a}$ & $33,33 \mathrm{a}$ & $29,50 \mathrm{a}$ & $33,84 \mathrm{a}$ & $36,30 \mathrm{a}$ \\
$\mathrm{Zn}$ & $10,39 \mathrm{a}$ & $9,05 \mathrm{ab}$ & $7,23 \mathrm{~b}$ & $9,01 \mathrm{ab}$ & $9,35 \mathrm{ab}$ \\
\hline
\end{tabular}

${ }^{1}$ Médias seguidas de mesma letra nas colunas não diferem estatisticamente, pelo teste deTukey $(P \geq 0,05) . n=5$.

Considerando-se a produção de grãos por unidade de nutriente aplicado (N, P e K), definida por FAGERIA (1998) como eficiência agronômica, verifica-se que os tratamentos T2 e T3 apresentaram os menores valores (Tabela 6). Isso ocorreu porque as plantas desses tratamentos apresentaram menor produção de grãos, em função dos estresses salinos aplicados durante do todo o ciclo (T2) ou na fase de crescimento inicial (T3), e as quantidades aplicadas de $\mathrm{N}, \mathrm{P}$ e $\mathrm{K}$ foram similares em todos os tratamentos.

A redução na eficiência agronômica na aquisição de nutrientes é relevante, visto que parte dos nutrientes aplicados não são utilizados pela cultura e ficam sujeitos ao processo de lixiviação. Vale salientar que o T3 apresentou forte crescimento vegetativo, de modo que a eficiência com base na produção primária $\left(E N_{P}\right)$ permaneceu elevada. Os resultados também sugerem que as adubações em culturas sob estresse salino podem ser realizadas com menores quantidades de fertilizantes (MARSCHNER, 1995; GRATTAN \& GRIEVE, 1999; LACERDA, 2005). As plantas dos tratamentos T1, T4 e T5 apresentaram elevadas eficiências agronômicas de N, o que se deve ao fato de que grande parte desse nutriente é adquirida na associação das raízes do feijão-de-corda com as bactérias fixadoras de nitrogênio (VASCONCELOS et al., 1987; RUMJANEK et al., 2005).

TABELA 6. Eficiência agronômica, kg de grãos produzidos/kg nutriente aplicado, em plantas de feijão-de-corda irrigadas com água salina em diferentes estádios de desenvolvimento. Nutrient use efficiency, kg of grains produced/kg applied nutrient in cowpea plants irrigated with saline water at different growth stages.

\begin{tabular}{cccc}
\hline \multirow{2}{*}{ Tratamentos } & \multicolumn{3}{c}{ Eficiência Agronômica } \\
\cline { 2 - 4 } & $\mathrm{N}$ & $\mathrm{P}$ & $\mathrm{K}$ \\
\hline 1 & 93,3 & 31,1 & 62,2 \\
2 & 49,2 & 16,4 & 32,8 \\
3 & 62,1 & 20,7 & 41,4 \\
4 & 91,3 & 30,4 & 60,8 \\
5 & 93,8 & 31,3 & 62,5 \\
\hline
\end{tabular}

*Os valores são médias conjuntas das cinco repetições.

\section{CONCLUSÕES}

A irrigação com água salina na germinação e na fase inicial de crescimento da cultura (T3) reduz a eficiência de utilização da maioria dos nutrientes, considerando-se a produtividade de grãos. 
A aplicação contínua de água salina (T2) reduz a eficiência agronômica de utilização de nutrientes, mas não afeta a eficiência de utilização dos nutrientes extraídos pelas plantas.

Os tratamentos T4 e T5 resultaram em maior economia de água de baixa salinidade, sem afetar a produtividade e as eficiências de utilização de água e nutrientes.

A estratégia de uso de água salina durante os estádios de desenvolvimento do feijão-de-corda pode contribuir para a obtenção de colheitas rentáveis e para diminuir o consumo de água de boa qualidade, devendo-se levar em consideração a maior sensibilidade da cultura na fase inicial do seu desenvolvimento.

\section{AGRADECIMENTOS}

Ao Fundo Setorial CT-HIDRO e ao Conselho Nacional de Desenvolvimento Científico e Tecnológico-CNPq, pelo suporte financeiro.

\section{REFERÊNCIAS}

ADEKALU, K.O.; OKUNADE, D.A. Effect of irrigation amount and tillage system on yield and water use efficiency of cowpea. Communications in Soil Science and Plant Analysis, Philadelphia, v.37, n.1/2, p.225-237, 2006.

AJEIGBE, H.A.; MOHAMMED, S.G.; SINGH, B.B. Comparative assessment of yield potentials of improved cowpea breeding lines using performance index and ranking methods. Journal of Food Agriculture and Environment, Helsinki, v.4, n.1, p.95-98, 2006.

ANDRADE, E.M.; PALÁCIO, H.A.Q.; SOUZA, I.H.; LEÃO, R.A.O.; GUERREIRO, M.J. Land use effects in groundwater composition of an alluvial aquifer (Trussu River, Brazil) by multivariate techniques. Environmental Research, Amsterndam, v.106, n.1, p.170-177, 2008.

ASSIS JÚNIOR, J.O. de; LACERDA, C.F.; SILVA, F.B. da; SILVA, F.L.B. da; BEZERRA, M.A; GHEYI, H.R. Produtividade do feijão-de-corda e acúmulo de sais no solo em função da fração de lixiviação e da salinidade da água de irrigação. Engenharia Agrícola, Jaboticabal, v.27, n.3, p.702713, 2007.

AYERS, R.S.; WESCOT, D.W. A qualidade da água na agricultura. Campina Grande: UFPB, 1999.153 p. (Estudos FAO, Irrigação e Drenagem, 29).

CHAUHAN, C.P.S.; SINGH, R.B. Suplemental irrigation of wheat with saline water. Agricultural Water Management, Amsterdam, v.95, n.3, p.253-258, 2008.

DADSON, R.B.; HASHEM, F.M.; JAVAID, I.; JOSHI, J.; ALLEN, A.L.; DEVINE, T.E. Effect of water stress on the yield of cowpea [Vigna unguiculata (L.) Walp] genotypes in the Delmarva region of the United States. Journal of Agronomy \& Crop Science, Malden, v.191, n.2, p.210-217, 2005.

DANTAS, J.P.; MARINHO, F.J.L.; FERREIRA, M.M.M.; AMORIM, M.S.N.; ANDRADE, S.I.O.; SALES, A.L. Avaliação de genótipos de caupi sob salinidade. Revista Brasileira de Engenharia Agrícola e Ambiental, Campina Grande, v.6, n.3, p.425-430, 2002.

FAGERIA, N.K. Otimização da eficiência nutricional na produção das culturas. Revista Brasileira Engenharia Agrícola e Ambiental, Campina Grande, v.2, n.1, p.6-16, 1998.

FERNANDES, V.L.B. (Coord.). Recomendações de adubação e calagem para o Estado do Ceará. Fortaleza: UFC, 1993. 248 p.

FREIRE FILHO, F.R.; RIBEIRO, V.Q.; BARRETO, P.D.; SANTOS, A.A. dos. Melhoramento genético. In: FREIRE FILHO, F.R.; LIMA, J.A. de A.; RIBEIRO, V.Q. (Ed.). Feijão caupi: avanços tecnológicos. Brasília: EMBRAPA, 2005. cap. 1. p 29-92. 
GHEYI, H.R. Problemas de salinidade na agricultura irrigada. In: OLIVEIRA, T.S.; ASSIS JÚNIOR, R.N.; ROMERO, R.E.; SILVA, J.R.C. (Eds.). Agricultura, sustentabilidade e o semiárido. Viçosa-MG: Sociedade Brasileira de Ciências do Solo, 2000. p.329-346.

GLENN, E.; TANNER, R.; MIYAMOTO, S.; FITZSIMMONS, K.; BOYER, J. Water use, productivity and forage quality of the halophyte Atriplex nummularia grown on saline waste water in a desert environment. Journal of Arid Environments, Amsterdam, v.38, n.1, p.45-62, 1998.

GRATTAN, S.R.; GRIEVE, C.M. Salinity-mineral nutrient relations in horticultural crops. Scientia Horticulturae, Amsterdam, v.78, n.1, p.127-157, 1999.

LACERDA, C.F. Interação salinidade x nutrição mineral. In: NOGUEIRA, R.J.C.; ARAÚJO, E.L.; WILLADINO, L.G.; CAVALCANTE, U.M.T. (Eds.). Estresses ambientais: danos e benefícios em plantas. Recife: UFRPE: Imprensa Universitária, 2005. p.95-105.

LACERDA, C.F.; ASSIS JÚNIOR, J.O.; LEMOS FILHO, L.C.A.; GUIMARÃES, F.V.A.; OLIVEIRA, T.S.; GOMES FILHO, E.; PRISCO, J.T.; BEZERRA, M.A. Morpho-physiological responses of cowpea leaves to salt stress. Brazilian Journal of Plant Physiology, Brasília, v.18, p.455-465, 2006.

MAAS, E.V. Testing crops for salinity tolerance. In: MARANVILLE, J.W.; BALIGAR, B.V.; DUNCAN, R.R.; YOHE, J.M. (Eds.). Workshop on Adaptation of Plants to Soil Stresses. INTSORMIL. Lincoln: University of Nebraska, 1993. p.234-247.

MAAS, E.V.; POSS, J.A. Salt sensitivity of cowpea at various growth stages. Irrigation Science, Heidelberg, v.10, n.4, p.313-320, 1989.

MALASH, N.; FLOWERS, T.J.; RAGAB R. Effect of irrigation systems and water management practices using saline and non-saline water on tomato production. Agricultural Water Management, Amsterdam, v.78, n.1, p.25-38, 2005.

MALAVOLTA, E.; VITTI, G.C.; OLIVEIRA, S.A. Avaliação do estado nutricional das plantas: princípios e aplicações. Piracicaba: POTAFÓS, 1997. 319 p.

MARSCHNER, H. Mineral nutrition of higher plants. London: Academic Press, 1995. 674 p.

MURILLO-AMADOR, B.; DIÉGUEZ, E.T.; HERNÁNDEZ, J.L.G.; AGUILAR, R.L; SERRANO, N.Y.A.; SALGADO, S.Z.; PUENTE, E.O.R.; KAYA, C. Effect of NaCl salinity in the genotypic variation of cowpea (Vigna unguiculata) during early vegetative growth. Scientia Horticulturae, Amsterdam, v.108, n.4, p.423-431, 2006.

MURTAZA, G.; GHAFOOR. A.; QADIR, M. Irrigation and soil management strategies for using saline-sodic water in a cotton-wheat rotation. Agricultural Water Management, Amsterdam, v.81, n.1, p.98-114, 2006.

NIELSEN, C.L.; HALL, A.E. Responses of cowpea (Vigna unguiculata (L.) Walp.) in the field to high night air temperature during flowering. II. Plant responses. Fields Crops Research, Amsterdam, v.10, p.181-196, 1985.

OSTER, J.D.; HOFFMAN, G.J.; ROBINSON, F.E. Management alternatives: crops, water, and soil. California Agriculture, Oak Ridge, v.36, n.1, p.29-32, 1984.

RHOADES, J.D.; KANDIAH, A.; MASHALI, A.M. Uso de águas salinas para produção agrícola. Campina Grande: UFPB, 2000. 117 p. (Estudos FAO. Irrigação e Drenagem, 48).

RUMJANEK, N.G.; MARTINS, L.M.V.; XAVIER, G.R.; NEVES, M.C.P.; FREIRE, E.A.; CAJAZEIRAS, J.B.; ANDRADE NETO, M.; GRANJEIRO, M.B.; CAVADA, B.S. Composição química da semente. In: FREIRE FILHO, F.R.; LIMA, J.A. de A.; RIBEIRO, V.Q. Feijão caupi: avanços tecnológicos. Brasília: EMBRAPA, 2005. cap. 8, p.339-365. 
SHARMA, D.P.; RAO, K.V.G.K. Strategy for long term use of saline drainage water for irrigation in semi-arid regions. Soil \& Tillage Research, Amsterdam, v.48, n.4, p.287-295, 1998.

SOUSA, M.F.; CAMPOS, F.A.P.; PRISCO, J.T.; ENÉAS-FILHO, J.; GOMES-FILHO, E. Growth and protein pattern in cowpea seedlings subjected to salinity. Biologia Plantarum, Praha, v.47, n.3, p.341-346, 2003.

SOUZA, M. do S.M.; BEZERRA, F.M.L.; TEÓFILO, E.M. Coeficientes de cultura do feijão caupi na região litorânea do Ceará. Irriga, Botucatu, v.10, n.3, p.241-248, 2005.

TAIZ, L.; ZEIGER, E. Plant physiology. 3.ed. Massachusetts: Sinauer Associates, 2002. 690 p.

VASCONCELOS, I.; MENDES FILHO, P.F.; ALMEIDA, R.T. Nodulação e desenvolvimento do feijão-de-corda, Vigna unguiculata (L) Walp., em solos com diferentes níveis de salinidade. Ciência Agronômica, Fortaleza, v.18, n.1, p.125-130, 1987.

WILSON, C.; LIU, X.; LESCH, S.M.; SUAREZ, L. Growth response of major USA cowpea cultivars: Biomass accumulation and salt tolerance. HortScience, Alexandria, v.41, n.2, p.225-230, 2006. 